\title{
Identifying Various Roadways Obstacles in Infrastructure less Environment Using Depth Learning Approach
}

\author{
CHANDRA KISHOR PANDEY ${ }^{1}$, NEERAJ KUMAR ${ }^{2}$, \\ VINAY KUMAR MISHRA ${ }^{3}$ and ABHISHEK BAJPAI ${ }^{4}$ \\ ${ }^{1,2,4}$ Department of Computer Application,Shri Ramswaroop Memorial University, \\ Deva Road, Barabanki, Uttar Pradesh, India. \\ ${ }^{3}$ Department of Computer Application,Shri Ramswaroop Memorial Group of \\ Professional Colleges, Lucknow, Uttar Pradesh, India.
}

\begin{abstract}
Traffic conditions in infrastructure-less environment are in many ways not ideal for driving. This is due to undefined road curvature, faded and unmaintained lane markings and various obstacles situations cause vital life loses and damage of vehicles in accidents. This paper provides an efficient approach of finding various roadways obstacles situation using our depth learning approach based on the data collected through a Smartphone. The existing methods are suitable for planned or structured roads. The proposed approach is suitable for planed as well as unplanned roads i.e. for infrastructure-less environment. The approach is capable of effectively classifying roadways obstacles into predefined categories using depth learning approach. While compared with other similar approach this approach is a cost effective approach.
\end{abstract}

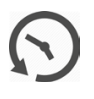

Article History

Received: 30 May 2017 Accepted:12 July 2017

\section{Keywords}

Smartphone, accelerometer, global positioning system, actionable obstacles, non-actionable obstacles, advanced driver assistance system.

\section{Introduction}

The automobile is one of the greatest inventions that have become an essential part of our daily activities. However, it can bring about disaster and can even kill us through accidents. Vehicle accident is a vital problem in today's world. Many a time, accidents and unusual traffic congestion take place due to careless and impatient nature of drivers. In most case drivers don't follow traffic lane, traffic signs, traffic rules leading to traffic congestion and accidents. As per National Crime Report Bureau (NCRB) report in 2016 there were high increases of road accidents cause un-natural death ${ }^{3}$. From last decade, Safety plays a major role in automobile industry, which results in the invention of various safety measures such as air bags, central locking system, automatic breaking system, traffic sign detection etc. Increasing the safety and saving

CONTACT Neeraj Kumar neeraj.cs@gmail.com 9 Department of Computer Application,m Shri Ramswaroop Memorial University, Deva Road, Barabanki, Uttar Pradesh, India.

(C) 2017 The Author(s). Published by Enviro Research Publishers

This is an $\bigcirc$ Open Access article licensed under a Creative Commons Attribution-NonCommercial-ShareAlike 4.0 International License (https://creativecommons.org/licenses/by-nc-sa/4.0/), which permits unrestricted NonCommercial use, distribution, and reproduction in any medium, provided the original work is properly cited.

To link to this article: http://dx.doi.org/10.13005/ojcst/10.03.05 
lives of human beings is one of the basic features in Advanced Driver Assistance System (ADAS), a component in Intelligent Transportation System (ITS). The Advanced Driver Assistance Systems (ADAS) has capability of decision making which assist drivers to make easy of vehicle driving process and it reduces the chances of accidents on the road. But, ADAS systems are costly hence all roadways vehicles owners cannot afford them. ADAS systems require training and maintenance which is rarely practiced by vehicle owners and finally due to unusual traffic situations on the roads, it is difficult to use advance driver assistance system in infrastructure less environment. A driver feels uncomfortable to control the roadways vehicles due to various roadways obstacles situations such as potholes, bumps, etc. in infrastructure less environment. For counter measuring all these problems, Roadway Obstacles Detection \& Warning System (RODWS) is needed that can assist people drive safely in that types of roads where no infrastructure is available and roads are not made on predefined rules in structured way. In this paper an efficient approach of finding various roadways obstacles situations in infrastructurefree environment is given where data is captured using Smartphone accelerometer and GPS. The approach is also capable to categories different obstacles situations in predefined categories.

\section{Various Roadways Obstacles Situations In Infrastructure-less Environment}

Roadways obstacles increase the likelihood of accidents and damage of vehicles. In rural area, in particular, roadways obstacles are a recurring problem. To find road obstacles in an automated and cost-effective manner, this paper proposes an efficient method for finding and classifying various obstacles situations in infrastructure-free environment. To collect data from the road, a Smartphone accelerometer and GPS is used in infrastructure less environment with the help of Smartphone's application. This information can adequately describe and locate roadways obstacles as a Smartphone-carrying vehicle drives through the roads. If we talk about vehicles then some vehicles may be public vehicles (e.g., buses, police cars) but most of the vehicles are driven by drivers. We use the term obstacles in a generic sense to describe various obstacles which include potholes, sunk castings, utility patches, catch basins, train tracks and speed bumps etc. The roadways obstacles mostly cause damage to the wheels or other parts of a vehicle. The raw data collected by Smartphone includes following two types of roadways obstacles:

- Actionable obstacles

- Non-actionable obstacles

\section{Actionable obstacles}

Actionable are obstacles which are caused by nature, through accidents on the roads or human made which require prompt attention. Following are some types of actionable obstacles found on unplanned roads or in infrastructure less environment:

\section{Potholes}

It is natural or human created hole on the road. It may be also caused due to wear and tear situation of the roads. It is severely found in rural roads.

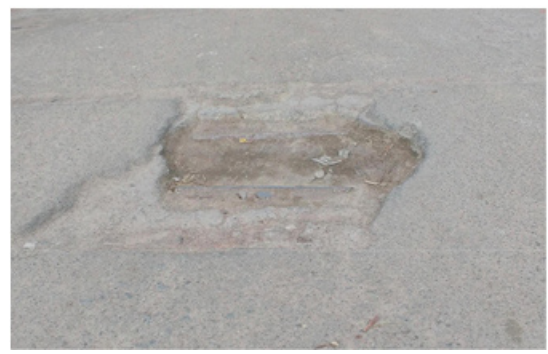

Fig. 1: Pothole

\section{Sunk castings}

These are manhole covers on the roads. It is mostly found in urban roads. These are made by mistakes of municipal corporation workers.

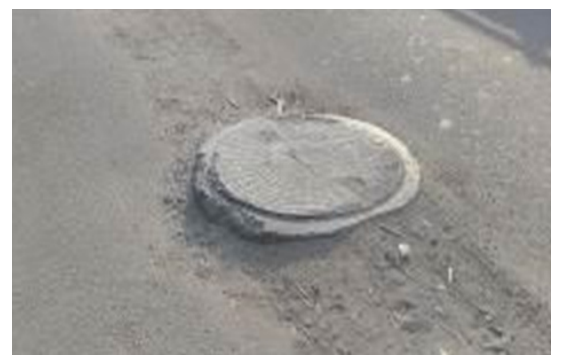

Fig. 2: Sunk casting 


\section{Utility patches}

In rural and urban roads at some places, roads are kept some high to make slow-down of vehicles running on the roads. Sometimes these extra patches cause accidents.

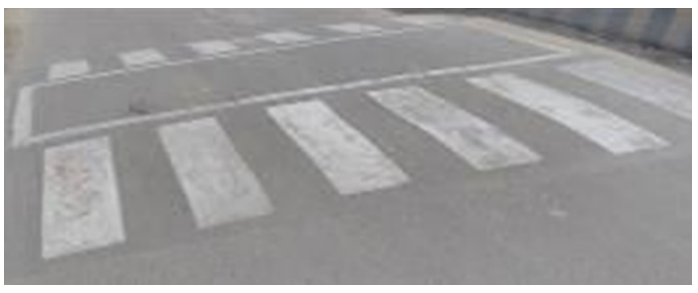

Fig. 3: Utility patch

\section{Catch basins}

These are cover of drains which mostly found in urban roads. These catch basin also cause accidents.

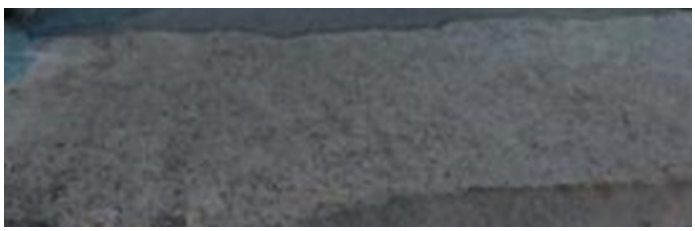

Fig. 4: Drains

\section{Speed bumps}

These are vehicle speed breakers through which vehicle speed can be reduced. Although NHAI recommended removing all road breakers from the highway but at some places basically in rural roads it found.

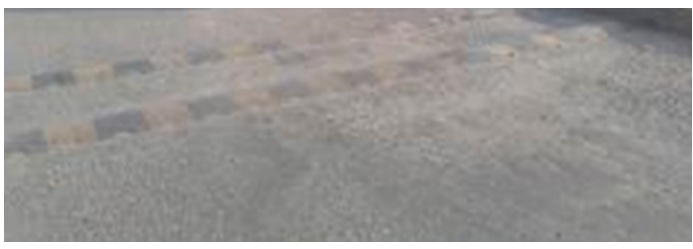

Fig. 5: Speed bumps

\section{Domestic animals sitting on the roads}

People rearing domestic animal keep free to wander here and there that's why the domestic animals used to sit on roadside cause accident.

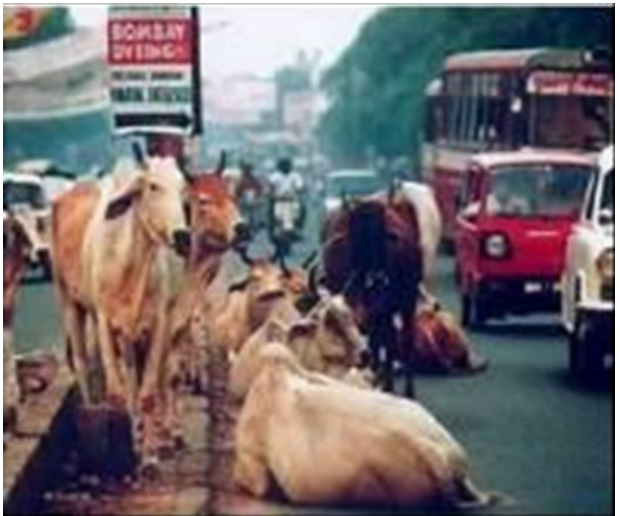

Fig. 6: Animals on the roads

\section{Construction material kept on roadside}

This habit is common basically in Indian roads. People don't bother to keep construction material on the roads cause road accidents.

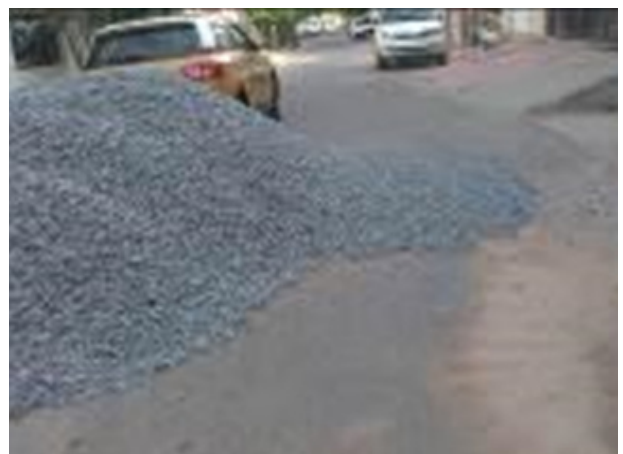

Fig. 7: Construction material kept on road

\section{People driving in wrong direction}

This is usual situations in rural roads but such situation can be also found in urban roads. People use to drive vehicle in wrong direction and due to such carelessness accidents occurred.

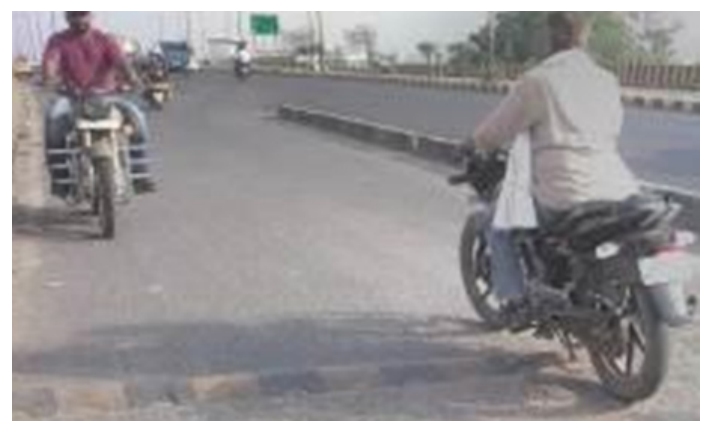

Fig. 8: People driving vehicles in wrong direction 


\section{Non-actionable obstacles}

These are such types of obstacles which do not require prompt attention and they are rarely responsible for roadways vehicle accidents. Such obstacles are not required for tracking all the times. Some non-actionable obstacles are as follows:

\section{Train tracks}

Train track comes under non-actionable obstacles because such obstacles are made as per requirement. Without train track it is not possible to run trains and roads where intersect train tracks are made at level of train track. It is also possible to be more than a pair of train track at a road intersection.

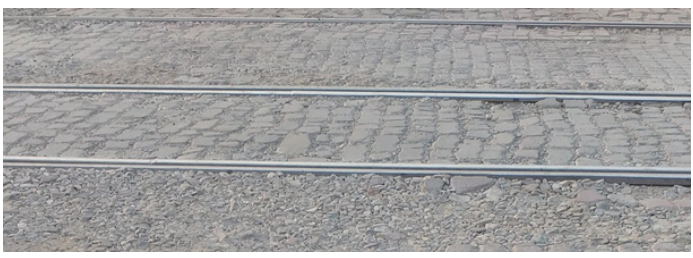

Fig. 9: Train tracks

\section{Relatively flat castings}

In current scenario roads have moderate highways and to show interconnection of two or more than two slips casting is made to join two or more than two slips. Such types of flat casting comes under the category of non-actionable obstacles.

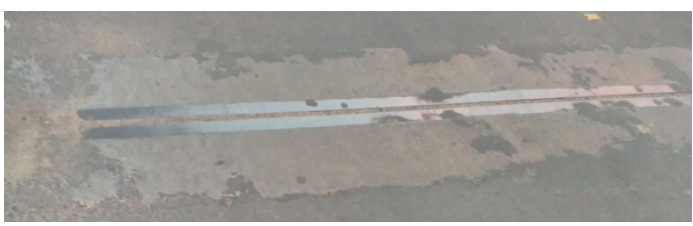

Fig. 10: Flat castings

\section{Related Work}

Nguyen Vinh Dinh et al. have proposed learning framework for robust obstacle detection, recognition, and tracking. In this paper they have introduced a general framework for detection, recognition and tracking preceding vehicles and pedestrian based on a deep learning approach. The proposed framework combines a novel deep learning approach with the use of multiple sources of local pattern and depth information to yield robust on-road vehicle and pedestrian detection, recognition and tracking ${ }^{1}$. Christos G. C. et al., have proposed a technique to find and categories various obstacles in planned roads. They have developed this method which collect data using Smartphone sensors and using iPhone application named as Speed Bump sense, classifying various obstacles on the road in smart cities. In their approach there is provision for anomaly detection and identification based on data collected using Speed Bump application. This method classifies the obstacles in predefined categories by using machine learning algorithms ${ }^{2}$. Sinan Kaplan et al. have proposed driver behavior analysis for safe driving: a survey. This paper discusses and provides a comprehensive insight into well-established techniques for driver inattention monitoring and introduces the use of most recent and futuristic solutions exploiting mobile technologies such as Smartphone and wearable devices ${ }^{4}$. Nils Gageik et al., have proposed an algorithm for obstacle detection and collision avoidance for a UAV with complementary low-cost sensors. This paper demonstrates an innovative and simple solution for obstacle detection and collision avoidance of unmanned aerial vehicles optimized for and evaluated with quad-rotors ${ }^{5}$. M. Khare et al. in their paper vehicle identification in traffic surveillance - complex wavelet transform based approach. In this paper they have proposed a method for vehicle identification which identified the objects present in a scene of traffic surveillance into two classes: vehicle and non-vehicle. The proposed method uses Daubechies complex wavelet transform as a feature of object, because Daubechies complex wavelet transform is approximate shift-invariant and have better edge representation property as compared to real valued wavelet transform ${ }^{6}$. Ajit Danti et al., have proposed a novice method for real time road boundary detection and vehicle detection for Indian roads. In this paper authors tried to analyze a wider view of the path data and evaluate the complete features of road status using real time VSP with open source tool OpenCV. This full frame processing application requires a low-cost frame grabber and a Pentium -based computer system for on-line real-time operations ${ }^{7}$. Lucky Kodwani has proposed an algorithm for real time vehicle detection and tracking by getting information of license plate of the vehicles. He presented an 
automatic system for collecting various vehicle data and tracking the vehicle in cost effective manner. His proposed system have many important utility in the field of pattern recognition and machine vision which may ranges from deep security systems to common discipline and from vehicle parking to metro city traffic control. The proposed algorithm has complex features due to various natural or human made effects. His work is based on the objective to show a process that solves the working difficulties of vehicle identification for real world data. The steps which are being used in this approach, from video data collection to OCR are permitted to get an real time identification of vehicles license plates $^{8}$. Sensor networks offer many attractive low cost solutions to monitor environmental conditions. Few applications of Sensor networks are volcano monitoring, machine monitoring, animal tracking and vehicle traffic monitoring ${ }^{10}$.

\section{Proposed Approach}

The proposed depth learning approach includes three main stages. First, road features recording using Smartphone; Second, feature construction for getting different types of road attributes for obstacles identification; third, classifying the identified obstacles into various categories.

\section{Recording Of Road Features By Smartphone} In Google play store there in an app Road BUMP through which roadways obstacle can be collected data from mobile sensors (3-axes accelerometer and GPS). Vehicle driver open the Road Bump application and put their phone on the fix position in vehicles, the position is decided by vehicle driver such dashboard etc. When vehicle is driven through the streets, the app automatically uses the phone's accelerometer and GPS receiver and collect information regarding obstacles. Following information is recorded using phone accelerometer:

- Latitudinal location of obstacle.

- Longitudinal location of obstacle.

- Vehicle speed in meter per second.

- Course, which is found to relation of the vehicle at the time of the obstacle.

- The Smartphone accelerometer records the $x, y, z$ readings of the obstacles, the Smartphone accelerometer also records the obstacle's time-stamp. As per Smartphone settings, $x, y, z$ axis locates north, west and $z$ locates gravity. To get road features clearly and easily we rotate this coordinate system so that the $\mathrm{x}$-axis aligns with the driving

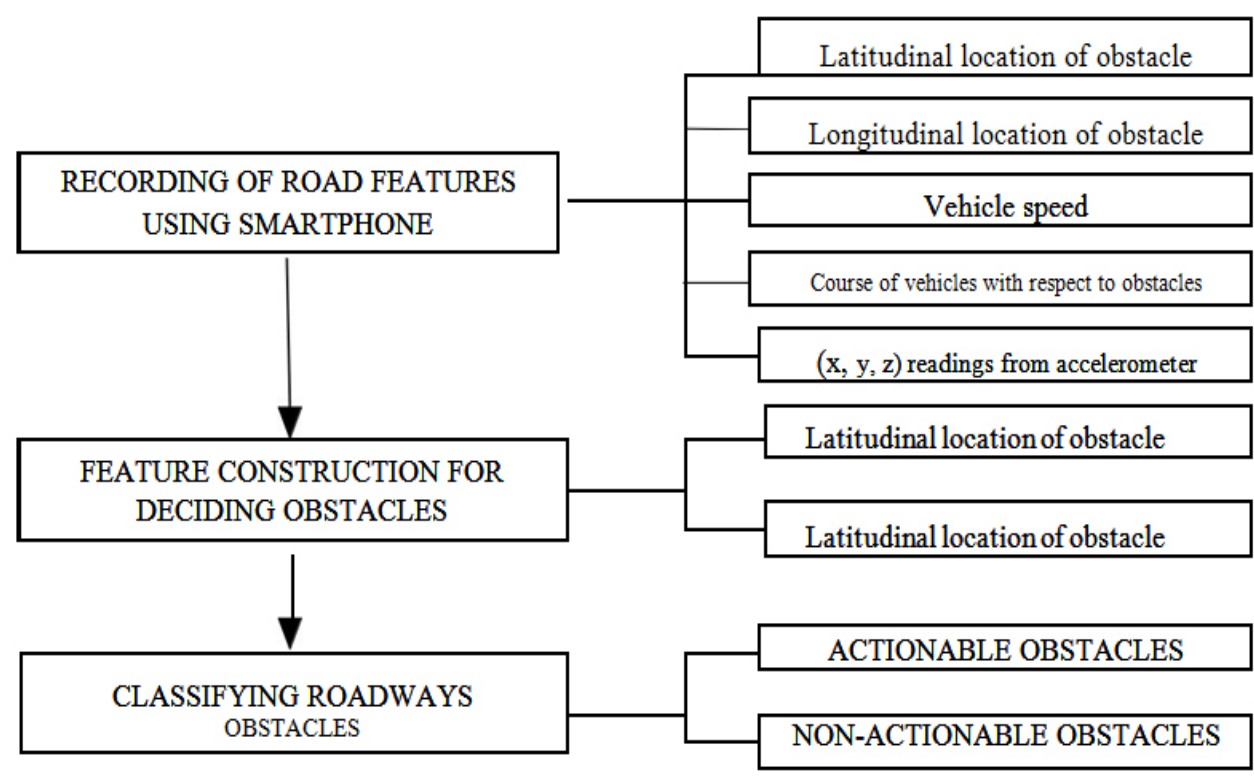

Fig. 11: Depth Learning Approach 
direction, the y-axis is perpendicular to it, and the z-axis is left unchanged.

\section{Feature Constrctuion For Deciding Obstacles}

After feature recording the next step of depth learning approach is feature construction of road data. In this step it is to be deciding whether the identified road features have obstacle situations or not. When the vehicle is on the road and speed of vehicle is greater than $14 \mathrm{~m} / \mathrm{s}$ and absolute value recorded using accelerometer is $0.4 \mathrm{~g}$ or above with related to z-axis then our algorithm proposed it obstacle.

The 3-dimensional coordinate time series data is equally distributed into $B$ bins . As all samples differ obstacle to obstacle, we divide each time series to manage an similar number of samples for every coordinate and for every roadway obstacle. In this step we derive the following attributes:

\section{Common obstacles attributes}

As we have mentioned earlier the common obstacles attributes may be latitudinal location and longitudinal location of the roadway obstacles and vehicle speed.

\section{Obstacles dispersal attributes}

From the set of obstacles attributes above, we also retain the obstacles dispersal features which state that how the roadways obstacles are dispersed with respect to the specific road.

\section{Classifying Roadways Obstacles Using Proposed Approach}

As we have mentioned in previous section, roadways obstacles are classified into two categories, i.e. actionable and non-actionable. For classifying obstacles in two groups, we pre-process the accelerometer measurements captured in the same vectors, $x, y$ and $z$ used earlier. After that observation of obstacles that simply check of the obstacles attributes because without attribute check it cannot be declared whether an obstacle is actionable obstacle or non-actionable obstacle. Following mathematical computation carried out for exact finding of whether an obstascles is actinable or non-actionable,
Let us assume $\gamma(\mathrm{k})$ the main values of accelerometer at sample time $\mathrm{k}$ referred to an obstacle(in either 3-axis). Similarly assume $\alpha(k)$ the amplitude difference calculated over two consecutive time steps:

$\alpha(k)=\gamma(k)-\gamma(k-1)$

The above calculation helps in finding difference between actionable and non-actionable obstacles but we have to enlarge the amplitude adding for more clear results.

In order to enlarges these amplitude adding, we again express a differentiate signal, which we termed to " $\beta$-signature filter". If the value of $\beta$-signature filter i.e. $\beta(k)$ is either greater than zero or less than zero then those obstacles is termed ad actionable obstacles otherwise the obstacles is termed as non-actionable obstacles.

$\beta(\mathrm{k})=\beta(\mathrm{k}-1)+\alpha(\mathrm{k})$, if $\alpha(\mathrm{k}) \alpha(\mathrm{k}-1)>0$

Here value of $\beta(k)$ is greater than zero, therefore in this situation it termed as actionable obstacles.

$\beta(k)=\alpha(k)$, if $\alpha(k) \alpha(k-1) \leq 0$

Here value of $\beta(k)$ is being no change, therefore in this situation it termed as non-actionable obstacles.

$\beta(k)=0$, if $\alpha(k) \alpha(k-1)<0$

Here value of $\beta(k)$ is being changed with respect to constant $\mathrm{c}$, therefore in this situation it termed as actionable obstacles. We can see that $\beta(\mathrm{k})$ either accumulates the adding $\alpha(\mathrm{k})$ if there is no change of sign, or it changes the value to the new difference otherwise.

The above approach works well in infrastructurefree environment for indentifying and classifying roadways obstacles. The methodology of finding obstacles situation is very small and easy therefore the proposed approach is cost effective. The approach is suitable for both types of roads: planned roads and unplanned roads i.e. in infrastructure-free environment. 


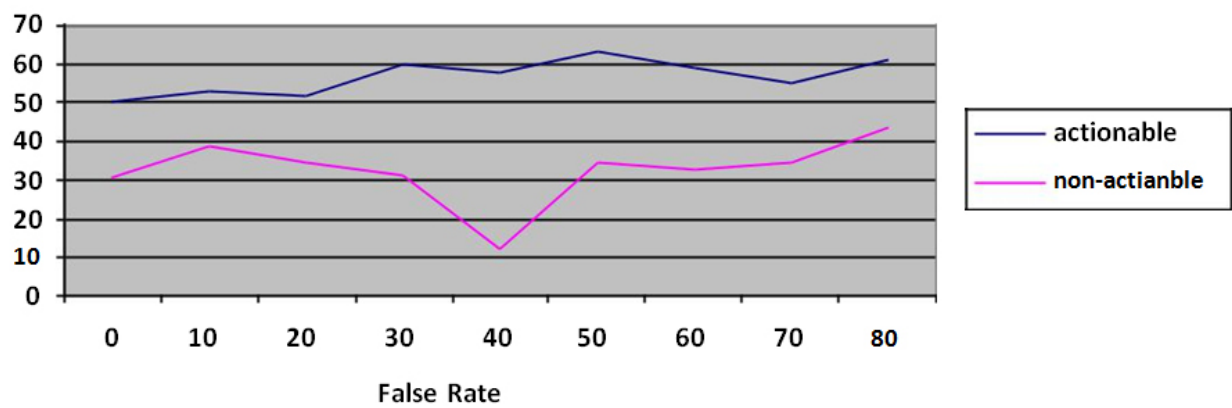

Fig.12: Comparative result of two types of obstacles

\section{Result Analysis}

Our results are totally depends upon an actual dataset with 105 roadways obstacles collected in infrastructure-free environment; we limit ourselves to reporting a top- $\mathrm{N}$ list of actionable obstacles. In section 3.2, as we have mentioned for each obstacle there are 40 to 60 samples are taken, and after that we have calculated the mode of division is 35 , while testing our algorithm, we adjust the value of $B$ to 4 , and $K$ to 6 . Total number of data used for binary classification is about to 80 . Finally we got that from above data set $52 \%$ of samples declared as actionable obstacles and $39 \%$ non-actionable obstacles. Our proposed approach gives accurate results for both types of obstacles situations i.e. for actionable obstacles and non-actionable obstacles as shown in figure 12.

\section{Conclusion}

In this paper we have proposed an approach for finding and classifying roadways obstacles into predefined categories which is useful for roadways vehicles. We have specified the high level design choices of the subsystem and justified the corresponding approach. The goal of this paper was to show how we can use the power of smartphone for driver support system. This approach is cost effective and can use this approach for developing an application running on android phone. In this paper we have concentrated on identifying and classifying obstacles situations in infrastructure free environment. The proposed algorithm is also useful for further research work in obstacle detection and tracking in infrastructure-free environment. In future we propose to do more experiments with variety of the situations.

\section{Reference}

1 Nguyen, Vinh Dinh, et al."'Learning Framework for Robust Obstacle Detection, Recognition, and Tracking." IEEE Transactions on Intelligent Transportation Systems 18.6 (2016): 1633-1646.

2 Brisimi, Theodora S., et al. "Sensing and Classifying Roadway Obstacles in Smart Cities: The Street Bump System." IEEE Access 4 (2016): 1301-1312.

3 RadhaKrishna, Kini A. "NCRB News Letter". http://ncrb.nic.in. N.p., 2016. Web. 30 May 2017.

4 Kaplan, Sinan, et al., "Driver behavior analysis for safe driving: a survey." IEEE Transactions on Intelligent Transportation
Systems 16.6 (2015): 3017-3032.

5 Gageik, Nils, Paul Benz, and Sergio Montenegro. "Obstacle detection and collision avoidance for a UAV with complementary low-cost sensors." IEEE Access 3 (2015): 599-609.

6 Khare, Manish, et al., "Vehicle Identification In Traffic Surveillance-Complex Wavelet Transform Based Approach." Journal of Science and Technology 52.4A (2014): 2938.

7 Danti, Ajit, and P. S. Hiremath. "A Realtime Road Boundary Detection and Vehicle Detection for Indian Roads", International Journal of Applied Information Systems 
(IJAIS), Volume 5- No.4, March 2013.

8

Kodwani, Lucky. "Automatic Vehicle Detection, Tracking and Recognition of License Plate in Real Time Videos." Communication and Signal Processing 2013 (2012).

9 Zhang, Kailong, et al., "Analysis and modeled design of one state-driven autonomous passing-through algorithm for driverless vehicles at intersections." Computational Science and Engineering (CSE), 2013 IEEE 16th International Conference on. IEEE, 2013.

10 Duvvuru, Santhosh Kumar. Design and deployment of vehicular traffic monitoring sensor networks. Diss. Indian Institute Of Technology-Bombay, 2008.

11 John Lu, Z. Q. "The elements of statistical learning: data mining, inference, and prediction." Journal of the Royal Statistical Society: Series A (Statistics in Society)
173.3 (2010): 693-694.

Cortes, Corinna, and Vladimir Vapnik. "Support-vector networks." Machine learning 20.3 (1995): 273-297.

13 Shawe-Taylor, John, and Nello Cristianini. Kernel methods for pattern analysis. Cambridge university press, 2004.

14 Bishop, Christopher M. "Pattern recognition." Machine Learning 128 (2006): 1-58.

15 Pudil, Pavel, Jana Novovièová, and Josef Kittler. "Floating search methods in feature selection." Pattern recognition letters 15.11 (1994): 1119-1125.

16 Arthur, David, and Sergei Vassilvitskii. "K-means++: The advantages of careful seeding." Proceedings of the eighteenth annual ACM-SIAM symposium on discrete algorithms. Society for Industrial and Applied Mathematics, 2007. 